LAWRENCE LIVERMORE NAT IO N A L LABORATORY

\section{A New Detailed Term Accounting Opacity Code: TOPAZ}

C. A. Iglesias, M. H. Chen, W. Isaacs, V. Sonnad, B. G. Wilson

May 4, 2004

Atomic Processes in Plasmas Santa Fe, NM, United States April 19, 2004 through April 22, 2004 
This document was prepared as an account of work sponsored by an agency of the United States Government. Neither the United States Government nor the University of California nor any of their employees, makes any warranty, express or implied, or assumes any legal liability or responsibility for the accuracy, completeness, or usefulness of any information, apparatus, product, or process disclosed, or represents that its use would not infringe privately owned rights. Reference herein to any specific commercial product, process, or service by trade name, trademark, manufacturer, or otherwise, does not necessarily constitute or imply its endorsement, recommendation, or favoring by the United States Government or the University of California. The views and opinions of authors expressed herein do not necessarily state or reflect those of the United States Government or the University of California, and shall not be used for advertising or product endorsement purposes. 


\title{
A New Detailed Term Accounting Opacity Code: TOPAZ
}

\author{
C. A. Iglesias, M. H. Chen, W. Isaacs, V. Sonnad, and B. G. Wilson
}

Lawrence Livermore National Laboratories

\begin{abstract}
A new opacity code, TOPAZ, which explicitly includes configuration term structure in the bound-bound transitions is being developed. The goal is to extend the current capabilities of detailed term accounting opacity codes such as OPAL that are limited to lighter elements of astrophysical interest. At present, opacity calculations of heavier elements use statistical methods that rely on the presence of myriad spectral lines for accuracy. However, statistical approaches have been shown to be inadequate for astrophysical opacity calculations. An application of the TOPAZ code will be to study the limits of statistical methods. Comparisons of TOPAZ to other opacity codes as well as experiments are presented.
\end{abstract}

\section{INTRODUCTION}

Recent improvements in opacity codes that include detailed term accounting (DTA) helped resolve several long-standing problems in astrophysics [1]. The main impact was due to the enhanced opacity from the forest of lines generated by the DTA calculations of $\mathrm{Fe}$ ions with partially filled M-shell configurations. Furthermore, OPAL calculations using full intermediate coupling together with astronomical observations and laboratory experiments suggest that pure LS coupling is insufficient for the iron group elements in astrophysical models $[1,2,3]$.

At present, however, astrophysical opacity codes [2,4,5] are limited to elements with atomic number less than about 30. For heavier elements DTA treatments are not only considered impractical but unnecessary. That is, the same myriad spectral lines that make the DTA approach unmanageable imply that statistical methods [6-8], which are computationally very fast compared to the DTA approach, should be reasonably accurate. Note, however, that statistical methods were shown to overestimate significantly astrophysical opacities [9].

The question then becomes: When do statistical methods for bound-bound transitions accurately describe the opacity of heavier elements? To address this issue the TOPAZ opacity code is being developed to extend the present DTA capabilities towards heavier elements. The resolution of this question should provide increased confidence on computed opacity databases; in particular, mid-Z elements that are used in radiation transport problems involving laboratory experiments. 


\section{THE TOPAZ CODE}

An important difference between opacity calculations for light and heavy elements is the immense number of atomic levels involved in the latter. Fortunately, computational methods and computer performance continue to improve. For example, parallel machines are becoming readily available where individual processors are considerably faster than any available when the light element opacity codes were developed. Also, the improved accuracy of $\mathrm{jj}$-coupling with increasing atomic number, thus avoiding the full intermediate coupling required for iron group elements, should significantly reduce the relative effort in the angular momentum coupling calculations.

The TOPAZ code takes advantage of these improvements and generates the necessary atomic data in-line; that is, it calculates the necessary atomic data (e.g.; energy levels and oscillator strengths) as required during the calculation. At present this option is desirable since the flexibility to change easily among different approximations using the same code far outweighs any additional cost in computational speed. In the future if many opacity calculations involving the same element at different matter conditions are to be performed (e.g.; an opacity table) the necessary atomic data could be generated at the start, stored, and read by subsequent calculations; thus, recovering any advantages derived from a database driven code.

The calculations are done in the single configuration approximation using a DiracHartree-Slater self-consistent field including Breit and QED corrections [10,11] as well as orbital relaxation effects. It is considered important to retain a full intermediate coupling capability. However, rather than performing the full intermediate coupling in the $j j$ basis, which requires limited configuration interaction calculations, an averaging procedure [12] of the relativistic results is employed followed by the full intermediate coupling in the LS representation.

The term structure in TOPAZ can be computed in a variety of approximations without affecting the central field results for the relativistic energies and radial integrals:

1) Neglect term structure

a) Non-relativistic configurations

b) Relativistic configurations

2) Statistical methods

a) Non-relativistic unresolved transition arrays [6]

b) Relativistic unresolved transition arrays [7]

3) Detailed term accounting

a) Pure LS coupling

b) Pure jj coupling

c) Full intermediate coupling in the LS basis

A major goal is to examine the limits of the statistical treatment to term structure by comparing results for a variety of elements and plasma conditions using the various approximations for the configuration term structure. Such comparisons will also help to identify and plan future experiments that can verify these theoretical studies.

By design the TOPAZ code does not compute the list of initial configurations necessary in opacity calculations. Instead, it reads the required information generated 
by other codes. This allows for direct comparisons of ionization balance calculations by different codes, including non-local thermodynamic equilibrium results, using the same photon absorption cross-sections.

\section{COMPARSIONS}

Although validation of opacity codes is essential, transmission experiments have proven difficult and not many are reported in the literature. Comparisons of TOPAZ to representative examples have been presented earlier along with discussions regarding the source of discrepancies between theory and experiments [13].

Here, a new comparison with a Ge experiment [14] performed at the NOVA Laser Facility is offered. The experiments took advantage of techniques developed to provide meaningful quantitative data on radiative properties of plasmas. For example, the temperature was measured to an accuracy of $\pm 4 \%$, mass density within $\pm 25 \%$, and transmission within \pm 0.025 . Furthermore, the samples were experimentally shown to be in local thermodynamic equilibrium with minimal gradients in density or temperature.

The spectral range studied in the experiments involved photon absorption from the L-shell of approximately 10 times ionized Ge ions. The instrumental spectral resolution was about $1.5 \mathrm{eV}$ to be applied to the theoretical transmission.

The comparisons shown in Ref. 14 were to the STA opacity code [8] that uses a statistical method based on the super-configuration concept. One conclusion [14] was the apparent overestimated absorption by theory of the $n=2$ to $n=3$ transitions. Line saturation effects and the experimental uncertainty in defining the zero transmission level, however, mitigate this discrepancy. More notable is the discrepancy in the $\mathrm{n}=2$ to $n=4$ arrays that clearly show narrower features in the model compared to the data. In this latter case saturation effects and experimental uncertainties due to background subtractions [14] are not expected to impact the results significantly.

TOPAZ calculations for the conditions in Ref. 14 were performed and compare to the experimental data as well as to other codes. The sample was atomically mixed plasma of equal parts $\mathrm{Al}$ and Ge by number at $38 \mathrm{eV}$ temperature and $0.012 \mathrm{~g} / \mathrm{cm}^{3}$ mass density. An initial super-configuration weighted list was generated by the VISTA code [15] that was later expanded into detailed non-relativistic configurations with initial probabilities. This latter list consisted of several million configurations but was reduced to 35006 by introducing an abundance cut-off greater than $10^{-6}$ for input into TOPAZ.

Preliminary tests were performed to determine the best choice of angular momentum coupling for the relevant Ge transitions. Here, the full intermediate coupling is assumed to be most correct but it is too time consuming for the complete calculation. Since the effect of spin-orbit splitting in these transitions is considerable, both LS coupling and unresolved non-relativistic unresolved transition arrays were found inadequate. On the other hand, $\mathrm{jj}$ coupling and relativistic unresolved transition arrays were found to be reasonably accurate; thus, the TOPAZ calculations for Ge displayed here use these two methods to account for the configuration term structure. 


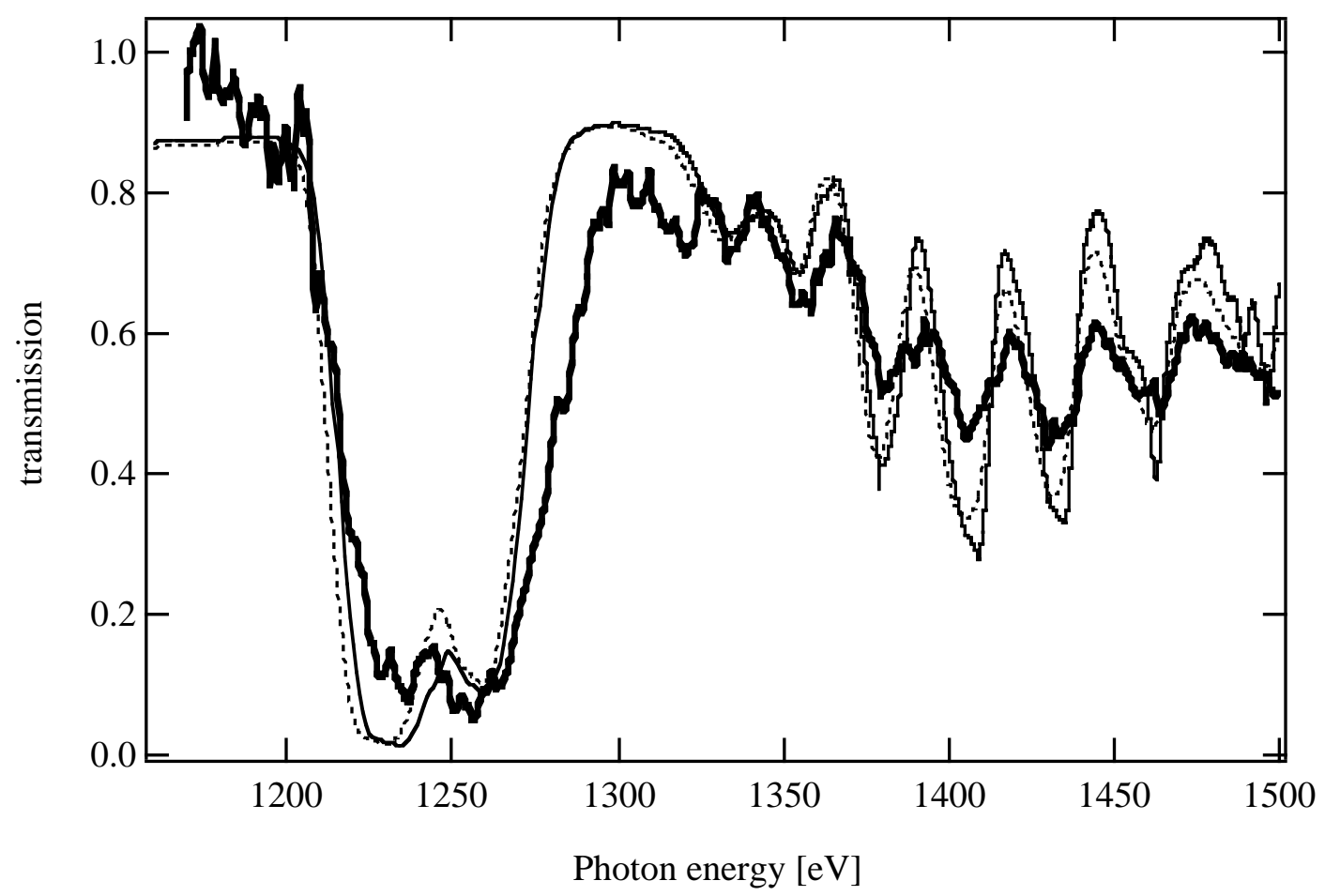

FIGURE 1. Comparison of experiment [14] (heavy solid), VISTA (dash), and TOPAZ/SOSA(solid).

Figure 1 shows a comparison of the transmission from experiment, VISTA, and TOPAZ using unresolved relativistic transition arrays or spin-orbit split arrays (SOSA) [7] over the full spectral range of the experiment. The comparison shows good agreement between VISTA and TOPAZ/SOSA but the discrepancy found earlier [14] with STA and experiment remains. The good agreement between the two models is welcomed since both use statistical methods for the bound-bound transitions and identical ionization balance.

Figure 2 and 3 compare the experiment and TOPAZ using DTA emphasizing the $\mathrm{n}=2$ to $\mathrm{n}=4$ spectral range. However, the TOPAZ results are convolved with a gaussian to simulate the experimental spectral resolution only in Fig. 3. In some cases it is important to compare the DTA results with and without the instrumental resolution function in order to demonstrate saturation effects. No such effect is apparent in this experiment and the impact of the resolution function on the transmission is minimal. Furthermore, since the discrepancies between experiment and theory remain, it suggests that the error is not caused by the statistical treatment of the bound-bound transitions but lies elsewhere. 


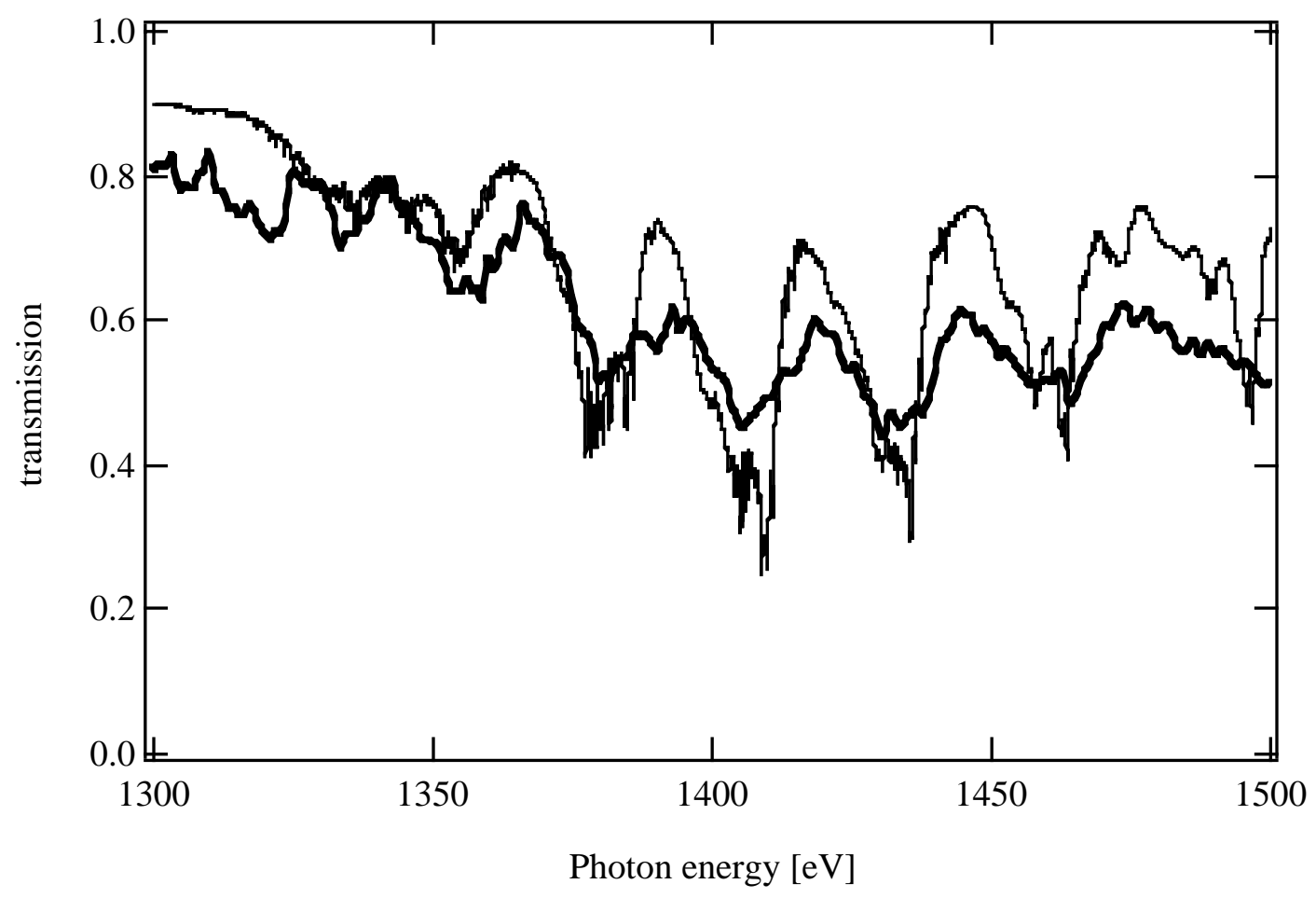

FIGURE 2. Comparison of experiment [14] (heavy solid) and TOPAZ/DTA(solid).

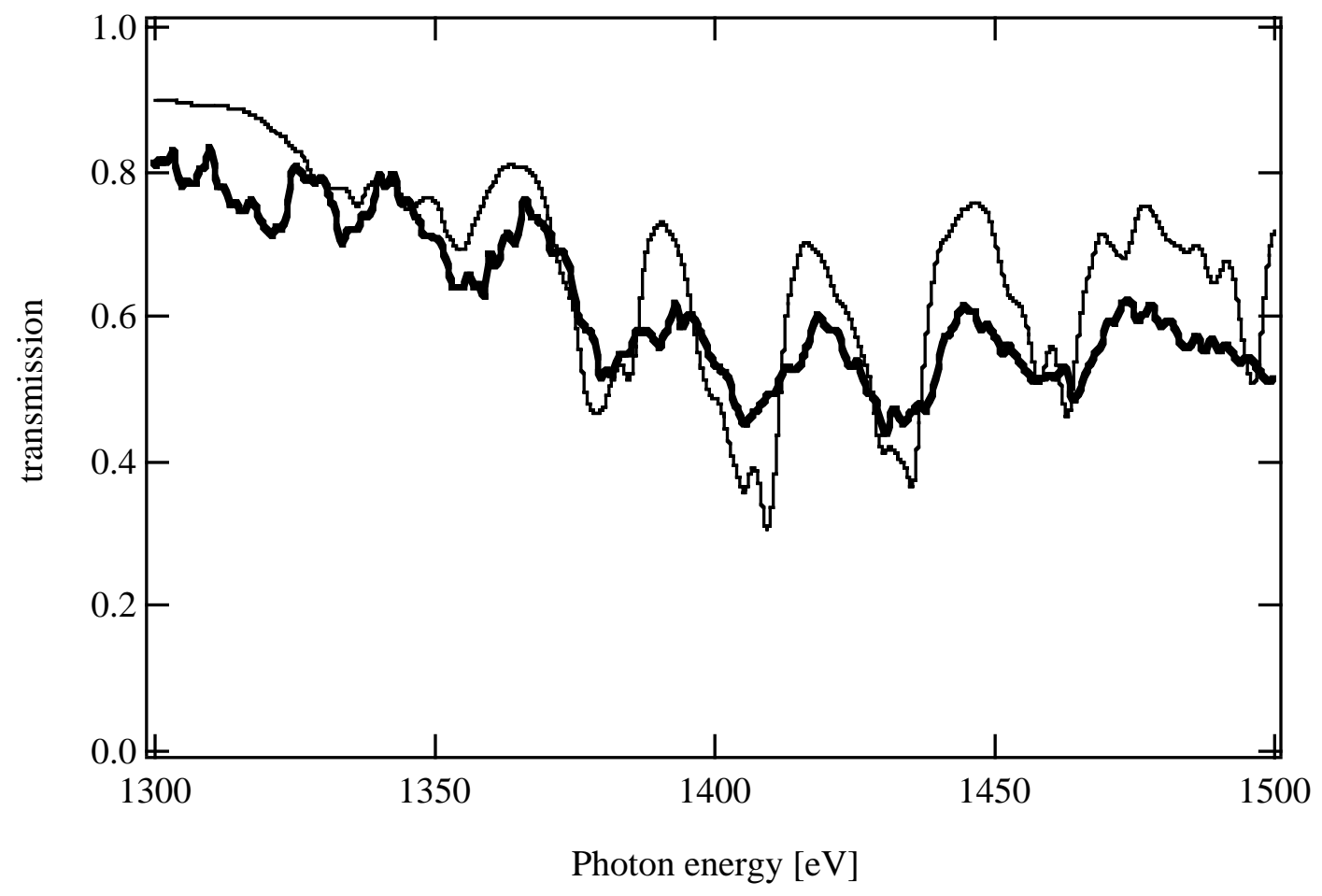

FIGURE 3. Comparison of experiment [14] (heavy solid) and TOPAZ/DTA (solid) convolved with experimental resolution. 
One possible explanation for the discrepancy may be an incorrect theoretical treatment of the spectral line widths. However, since autoionizing widths for these lines are expected to be smaller than $1 \mathrm{eV}$, it would require increases of the electron impact widths by more than an order of magnitude.

\section{PARALLEL TOPAZ CODE}

Although parallel machines provide large computational capabilities, it is important to take advantage of these resources efficiently. For example, an increase in the number of processors used in a given calculation should result in a comparable decrease in computational time. This proportional "speedup" indicates that very larger problems can then be addressed with massively parallel computers.

Opacity codes can readily take advantage of parallel architecture since many of the calculations involve independent processes. The parallel TOPAZ code initially did the calculations by performing all necessary photon absorption cross-sections for each initial non-relativistic configuration in an individual processor and the partial results accumulated to form the total opacity. Because different configurations require disparate computational effort, it resulted in an unbalanced workload and inefficient calculation. Presently, the code separates the calculation by sending each initial/final relativistic configuration pair of a bound-bound transition array to an individual processor providing considerable improvement in the load balance.

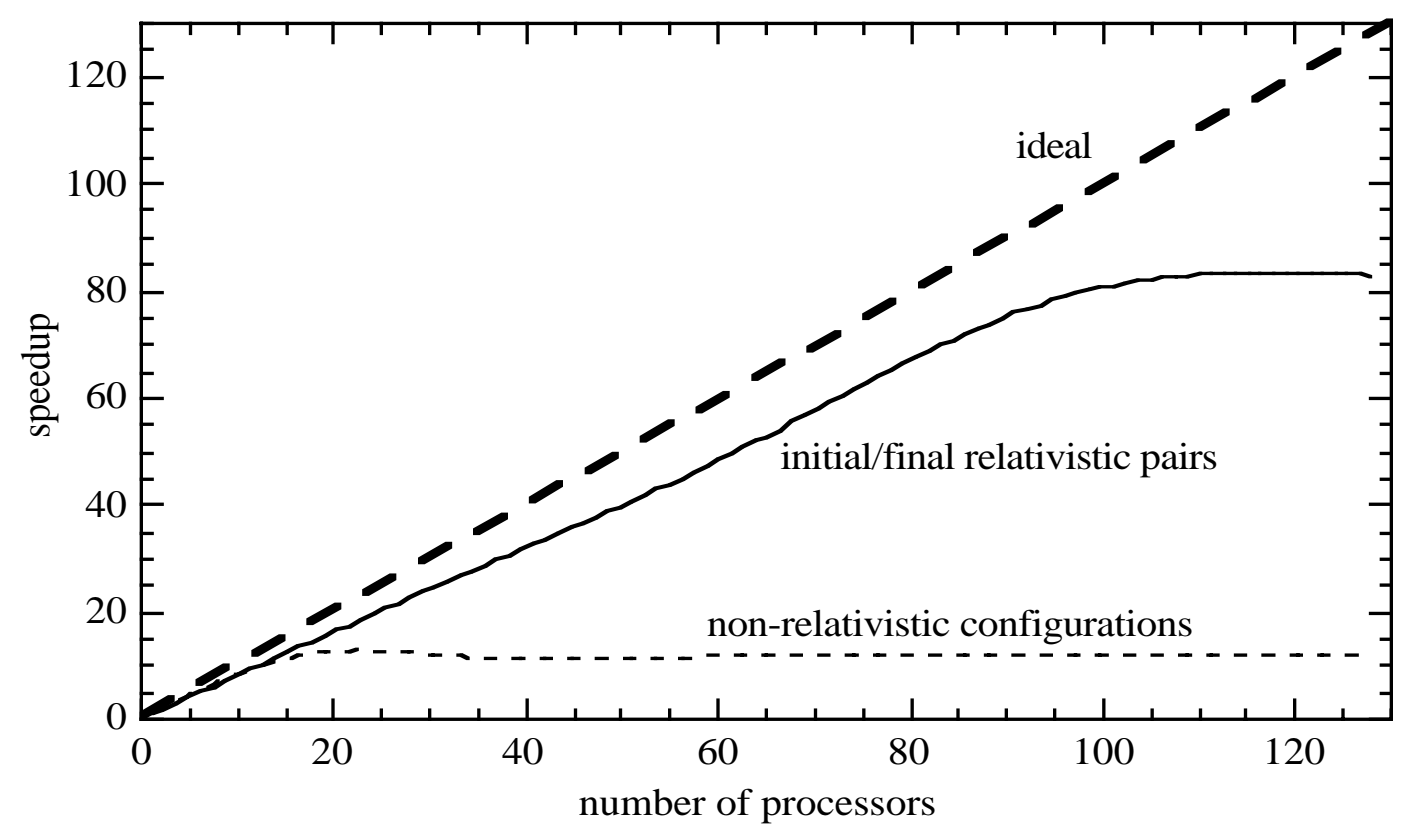

FIGURE 4. Comparison of the speedup for two parallel versions of TOPAZ with "ideal" representing the theoretical maximum.

The results in Fig. 4 demonstrate the improved efficiency by comparing the speedup for both parallel versions above. The results are for a reduced version of the AlGe calculations presented in Figs 1 to 3 using 1400 non-relativistic initial configurations. Preliminary testing suggests that to maintain the high efficiency the 
total number of processors should be less than about one tenth the number of initial non-relativistic configurations in a given calculation.

\section{CONCLUSION}

The TOPAZ opacity code has been developed and is, in principle, capable of computing opacities for heavy elements using detailed term accounting for the boundbound transitions. The code has been successfully tested against other opacity codes as well as experiments for elements as heavy as $\mathrm{Nb}$.

There remain several outstanding issues. Most important are discrepancies between experiment and theory, such as the one presented above for $\mathrm{Ge}$, which are shared by several opacity models and require further investigation or new experiments.

Specifically to TOPAZ, there is the question if jj-coupling is sufficiently fast and/or accurate for general opacity calculations of heavy elements. Nevertheless, identifying the range of validity of statistical methods for treatment of the bound-bound transitions in opacity codes remains a crucial objective. The ability of TOPAZ to compute the bound-bound transition in a variety of approximations is expected to play an important role towards this goal similar to that played by OPAL in the demonstration of not only the need for DTA but also full intermediate coupling of iron group elements for astrophysical opacities.

\section{ACKNOWLEDGMENTS}

Work performed under the auspices of the Department of Energy by Lawrence Livermore National Laboratory under Contract W-7405-ENG-48.

\section{REFERENCES}

1. F. J. Rogers and C. A. Iglesias, Science 263, 50(1997).

2. C. A. Iglesias, and F. J. Rogers, Ap. J. 464, 943(1996); and references therein.

3. C. A. Iglesias, F. J. Rogers, and B. G. Wilson, Ap. J. 397, 717(1992).

4. M. J. Seaton, Y. Yan, D. Mihalas, and A. K. Pradhan, MNRAS 266, 805(1994).

5. N. Magee et al, LANL Report LA-UR-97-1038.

6. C. Bauche-Arnoult, J. Bauche, and M. Klapsch, Phys. Rev. A20, 2424(1979); ibid, A25, 2641(1982)

7. C. Bauche-Arnoult, J. Bauche, and M. Klapsch, Phys. Rev. A31, 2248(1985)

8. A. Bar-Shalom et al, Phys. Rev. A40, 3183(1989)

9. C. A. Iglesias and B. G. Wilson, JQSRT 52, 127(1994).

10. D. A. Liberman, Phys. Rev. B2, 244(1970).

11. K. N. Huang et al, At. Data Nucl. Data Tables 18, 243(1976).

12. F. P. Larkins, J. Phys. B9, 37(1976).

13. C. A. Iglesias et al, JQSRT 81, 227(2003).

14. T. S. Perry et al, JQSRT 54, 317(1995).

15. VISTA is a relativistic LTE opacity code developed by M. H. Chen based on STA theory [8] using Dirac-Hartree-Slater methods with Breit and QED corrections for the atomic data. 